\title{
Coding of hedonic and nonhedonic samples by pigeons in many-to-one delayed matching
}

\author{
THOMAS R. ZENTALL and LOU M. SHERBURNE \\ University of Kentucky, Lexington, Kentucky \\ and \\ PETER J. URCUIOLI \\ Purdue University, West Lafayette, Indiana
}

\begin{abstract}
In two experiments, pigeons were trained on many-to-one delayed matching in which samples of food and one hue were each associated with one shape comparison, and samples of no food and a different hue were each associated with a second shape comparison. When later tested with delays between sample and comparison stimuli, pigeons showed nonparallel delay functions, typically found with food and no-food samples (i.e., steeply declining food-sample delay functions, and relatively flat no-food-sample delay functions). Furthermore, the slopes of the hue-sample delay functions were similar to those on the food/no-food-sample trials. In Experiment 2, following many-toone delayed matching, when the hue samples were associated with new comparisons and then food and no-food samples replaced the hues, evidence was found for transfer of training indicative of the common coding of samples associated with the same comparison in original training. The transfer results suggest that the asymmetrical hue-sample functions resulted from the common coding of samples associated with the same comparison.
\end{abstract}

In a conditional discrimination task, when two or more sample stimuli are each associated with reinforced responding to the same test or comparison stimulus (manyto-one matching) a relation may emerge between those samples. For example, if on some trials a red sample is associated with a circle comparison and on others a vertical sample is associated with the same circle comparison, evidence suggests that the two samples are commonly coded (see Urcuioli, Zentall, Jackson-Smith, \& Steirn, 1989).

The nature of this common code may be a representation of one of the samples (e.g., the vertical sample may be coded as the red sample; see, e.g., Zentall, Steirn, Sherburne, \& Urcuioli, 1991), a representation of the common correct comparison (e.g., both red and vertical samples may be coded as circle; Grant, 1991), or even a representation involving an arbitrary stimulus class (e.g., both samples may be coded as "stimulus A"; Grant, 1991).

Suggestive evidence for common coding in many-toone delayed matching-to-sample (MTO DMTS) by pigeons was first reported by Zentall, Urcuioli, Jagielo, and Jackson-Smith (1989). In that study, the effect on

This research was supported by National Science Foundation Grants BNS-8418275 and BNS-9019080 and National Institute of Mental Health Grant 45979 to T.R.Z. and P.J.U. The authors thank Karen L. Roper for her assistance. Correspondence should be addressed to T. $R$. Zentall, Department of Psychology, University of Kentucky, Lexington, KY 40506 (e-mail: zentall@ukcc).

-Accepted by previous editor, Vincent M. LoLordo delay performance of easy-to-discriminate hue samples was compared with that of hard-to-discriminate lineorientation samples (see, e.g., Farthing, Wagner, Gilmour, \& Waxman, 1977). Across groups, the sample/ comparison mapping was varied. In all mapping arrangements in which one sample was uniquely associated with one comparison (one-to-one mapping) or one sample was associated with each of two comparisons (one-tomany mapping), performance on hue-sample trials was considerably more accurate than performance on linesample trials. In the MTO DMTS group, however, the sample-dimension effect was virtually absent. Thus, it appears that the samples were coded differently by pigeons in the MTO DMTS group. Specifically, the data suggest that the samples associated with the same comparison were commonly coded, either as a representation of one of the samples, or in terms of a superordinate stimulus class. Although the common codes could have been prospective response intentions to peck the correct comparisons, this hypothesis is not supported by the fact that delayed matching performance did not depend on comparison discriminability (i.e., whether the comparisons were hues versus lines).

If MTO DMTS training results in common coding, one should be able to demonstrate these emergent relations in other ways. For example, if during MTO DMTS training, samples associated with the same comparison are commonly coded and one of those samples is then associated with a new comparison, one should be able to find evidence of an emergent relation between the new comparison and the remaining sample. To test this hypothesis, Urcuioli et al. (1989) trained pigeons to asso- 
ciate one pair of the original samples from an MTO DMTS task with a new pair of comparison stimuli. When the remaining pair of samples was then presented with the new comparisons, evidence of immediate transfer was found (see also Zentall, Sherburne, \& Urcuioli, 1993; Zentall et al., 1991, for other procedures that have been used to demonstrate common coding of samples in MTO DMTS).

The purpose of the present experiments was to attempt to identify the form of the common codes that develop in MTO DMTS. To accomplish this we used pairs of samples known to generate quite different patterns of retention functions. For example, presence/absence samples, such as food and no food, typically yield distinctive, nonparallel delay functions when delays are inserted between the sample and comparison stimuli (Colwill, 1984; Grant, 1991; Maki \& Hegvik, 1980; Maki, Olson, \& Rego, 1981; Sherburne \& Zentall, 1993b; Wilson \& Boakes, 1985). Performance on no-food-sample trials is generally high and shows little or no decrease as the delay increases, whereas performance on food-sample trials generally decreases rapidly with increasing delay. Such findings have been interpreted as evidence that the food-sample event is coded in memory and undergoes forgetting as the delay increases, whereas the no-foodsample event is not coded. Instead, a response to the comparison that is correct on no-food sample trials is a default response made only in the absence of memory of a food sample (see Colwill, 1984; Maki et al., 1981; Wilson \& Boakes, 1985).

On the other hand, hue samples (e.g., red and green), of the type commonly used in delayed matching research (see e.g., Berryman, Cumming, \& Nevin, 1963; Roberts, 1972; Zentall, 1973) typically yield delay functions that are moderately sloped and that are indistinguishable from each other. Thus, if hedonic and hue samples were intermixed in MTO DMTS, whether the resulting hue versus hedonic delay functions are symmetrical or not should help identify properties of the common codes.

In Experiment 1, we first trained pigeons to associate one member of a food/no-food (hedonic) sample pair and one member of a red-green sample pair with one of the comparisons and the two remaining samples with the other comparison. We then examined the delay functions. If representations of the hedonic samples formed the basis for common coding, one would expect both pairs of samples to yield distinctive, nonparallel delay functions. If, on the other hand, representations of the hue samples formed the basis for common coding, one would expect both pairs of samples to yield nondistinctive, parallel delay functions (e.g., Grant, 1991).

\section{EXPERIMENT 1}

\section{Method}

\section{Subjects}

The subjects were 12 mixed-sex experimentally naive White Carneaux pigeons purchased as retired breeders (over 5 years old) from the Palmetto Pigeon Plant (Sumter, SC). The pigeons were housed in individual cages with continuous access to water and grit. They were maintained throughout the experiment at $75 \%-80 \%$ of their free-feeding body weights. The colony room in which they were kept was maintained on a 12:12-h light:dark cycle.

\section{Apparatus}

The experiment was conducted in a sound-attenuated test chamber with a three-key response panel. The pigeon compartment measured $33 \mathrm{~cm}$ high $\times 31 \mathrm{~cm}$ wide $\times 35 \mathrm{~cm}$ across the response panel. The three rectangular pecking keys $(3.0 \mathrm{~cm}$ wide $\times 2.5 \mathrm{~cm}$ high) were mounted side by side $(.5 \mathrm{~cm}$ apart) on the panel with their bottom edges $21.0 \mathrm{~cm}$ from the wire mesh floor. Behind each pecking key was a 12-stimulus in-line projector (Industrial Electronics Engineering, Series 10 with General Electric No. 1820 lamps) that projected white (W, no filter) or red (R) or green $(\mathrm{G})$ hues (Kodak Wratten filters Nos. 26 and 60, respectively) on the center key and a white line-drawn circle (C, $16 \mathrm{~mm}$ outside diameter, $13 \mathrm{~mm}$ inside diameter) or a white dot (D, $5 \mathrm{~mm}$ diameter) on a black background, on the side keys. The opening $(6.0 \times 5.0 \mathrm{~cm})$ to three rear-mounted grain feeders was horizontally centered on the pecking panel and was located midway between the bottom of the pecking keys and the floor. Each of the three grain feeders could be independently raised to be accessible to the pigeon. One feeder was filled with mixed grain (Purina Pro Grains, the end-oftrial reinforcement), the second feeder was filled with green split peas (the food, F, sample), and the third feeder was weighted but remained empty (the no-food, NF, sample). Whenever one of the three feeders was raised, the feeder light was turned on.

A shielded houselight, located $5.0 \mathrm{~cm}$ above the top edge of the center key, provided general chamber illumination. White noise, at $72 \mathrm{~dB}$, and an exhaust fan provided masking noise. The experiment was controlled by a microcomputer located in an adjacent room.

\section{Procedure}

Pretraining. All pigeons were trained to eat from the mixedgrain feeder and were then shaped by the method of successive approximations to peck $\mathrm{W}$ on the center key. Each peck was reinforced. Once each bird pecked the center key 24 times, a single C or $D$ was presented on either the left or the right side key, and again a single peck was reinforced. Following each reinforcement there was a 3-sec intertrial interval (ITI) and the stimulus or the location of the stimulus changed. There was a total of 24 single-stimulus presentations, 6 of each stimulus (C and D), in each location (left and right), with the order randomly determined.

MTO DMTS training. On the day following pretraining, each pigeon began 0 -sec delay MTO DMTS training, with $F$ and one of the hues (referred to as the food hue, FH) associated with responding to one of the comparisons, and NF and the other hue (referred to as the no-food hue, NFH) associated with responding to the other comparison. The comparison associated with the $\mathrm{FH}$ and NFH sample and the hue that served as the FH and NFH sample were balanced over pigeons.

Each trial began with presentation of $\mathrm{W}$ on the center key. One peck to $W$ (which served as a ready signal) resulted in a 2-sec sample presentation (F, NF, R, or G), followed by comparison onset (C and $D$ ) on the side keys. A single response to the correct comparison resulted in 2-sec access to mixed grain as reinforcement and a 10-sec ITI. A single response to the incorrect comparison resulted in only the ITI. The houselight was lit only during the ITI. Sample stimulus and position of the correct comparison stimulus were counterbalanced across the 64 trials in each session. Trial types were randomly presented, with the constraint that on no more than three consecutive trials could either a particular comparison (C or D) or a particular comparison location (left or right) be correct.

Each pigeon was trained on this task either (1) to a performance criterion of $90 \%$ correct for two consecutive sessions, with performance on each of the four trial types at $81 \%$ correct or better, or 
(2) for 125 sessions. For all pigeons that did not reach criterion within 80 sessions, the duration of the samples was increased from $2 \mathrm{sec}$ to $4 \mathrm{sec}$ for the remainder of training, as well as during delay testing. Because all of the statistical tests involved within-subject comparisons, it was not anticipated that the increase in sample duration would adversely affect the conclusions that could be reached from the delay test. Sessions were conducted 6 days a week.

Delay test. Following acquisition, each pigeon received mixeddelay training involving equal numbers of each trial type with each of four delay intervals $(0,1,2$, and $4 \mathrm{sec})$ inserted between the offset of the sample and the onset of the comparisons. There was no chamber illumination during the delay. Delay testing involved a total of ten 64-trial sessions.

\section{RESULTS}

\section{Acquisition}

Seven of the 12 pigeons acquired the MTO DMTS task within 43 sessions and 1 pigeon took 114 sessions to reach criterion. Four pigeons failed to reach criterion within 125 sessions, but all of these pigeons matched at $81 \%$ correct on each of the four trial types. In all analyses performed on the data, the .05 level of significance was adopted.

Only 1 of the pigeons learned the hue-sample component of the task faster than the hedonic-sample component. Because there was considerable variability among the pigeons in sessions to criterion for the hedonic and hue samples, a sign test was used to compare acquisition of the two sample types. The sign test indicated that the hedonic-sample component of the task was learned significantly faster than the hue-sample component.

An analysis of variance (ANOVA) was performed on pecks to the FH sample versus pecks to the NFH sample, pooled over the last 3 days of training, with the hue of the sample ( $R$ vs. G) as a second factor. The analysis indicated that there was significantly more pecking to the NFH (49.2 pecks per session) than to the FH sample (39.7 pecks per session) $[F(1,10)=5.53]$. There was also significantly more pecking to the $G$ sample ( 50.3 pecks per session) than to the $\mathrm{R}$ sample (38.6 pecks per session) $[F(1,10)=8.26]$; however, the Hue $\times \mathrm{FH}$ versus $\mathrm{NFH}$ interaction was not significan $[F(1,10)=2.61]$.

\section{Delay Test}

Hedonic-sample trials. Delay performance (pooled over the 10 test sessions) on the two hedonic-sample trial types was similar to that reported by Sherburne and Zentall (1993b). Performance on F-sample trials was most accurate at the 0 -sec delay $(97.2 \%$ correct) and dropped sharply as the delay increased (to $54.8 \%$ correct at the 4 sec delay). On the other hand, accuracy of performance on NF-sample trials was somewhat lower at the 0 -sec delay $(80.4 \%$ correct) but dropped much less with increasing delays $(71.2 \%$ correct at the 4 -sec delay). An ANOVA performed on these data, with trial type ( $F$ vs. $\mathrm{NF})$ and delay $(0,1,2$, and $4 \mathrm{sec})$ as factors, indicated that although there was not a significant effect of trialtype $(F<1)$, there was a significant trial type $\times$ delay interaction $[F(3,33)=17.10]$. The effect of delay was, of

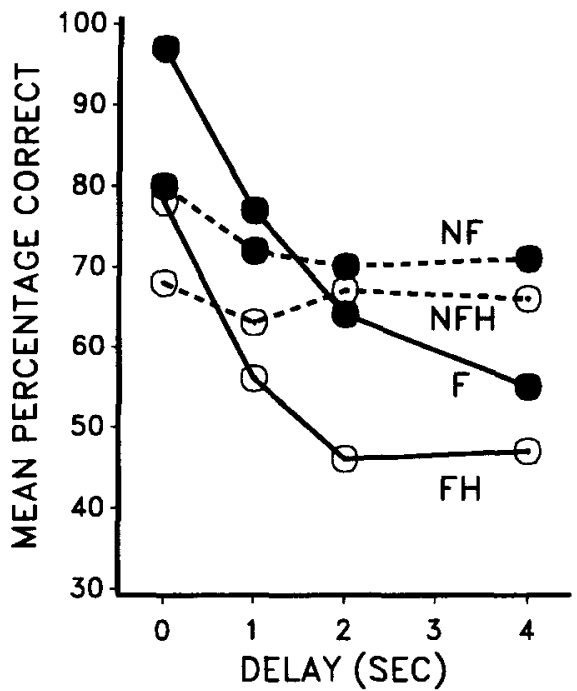

Figure 1. Experiment 1. Retention functions for each of the sample trial types in the many-to-one matching task: Food-sample trials (F), no-food-sample trials (NF), hue-sample trials for which the correct comparison was the same as on food-sample trials (FH), and hue-sample trials for which the correct comparison was the same as on no-food-sample trials (NFH).

course, also significant $[F(3,33)=58.16]$. The delay data for F- and NF-sample trial types appear in Figure 1.

Hue-sample trials. A similar pattern of results was found for FH- and NFH-sample trial types. Performance on the FH-sample trial type was relatively high at the 0 -sec delay ( $78.0 \%$ correct) but dropped sharply (to $46.8 \%$ correct at the 4-sec delay). On the other hand, performance on the NFH-sample trial type was not quite as high at the 0 sec delay (68.5\% correct), but dropped very little over delays (to $66.0 \%$ correct at the $4-\mathrm{sec}$ delay). An ANOVA performed on the hue-sample data indicated that matching accuracy on NFH-sample trials was not significantly higher $(66.1 \%$ correct $)$ than on FH-sample trials $(56.4 \%$ correct) $[F(1,11)=1.95]$, but once again, there was a significant trial type $\times$ delay interaction $[F(3,33)=6.96]$, as well as a significant delay effect $[F(3,33)=18.37]$.

All trials. The delay data from all sample trial types were combined in a three-way repeated measures ANOVA with sample type (hedonic vs. hue samples), common coding ( $\mathrm{F} / \mathrm{FH}$ vs. NF/NFH), and delay as factors. Consistent with the results of the analyses reported earlier, there was a significant common coding $\times$ delay interaction $[F(3,33)=11.11]$. More important, the three-way sample type $\times$ common coding $\times$ delay interaction was not significant $[F(3,33)=1.81]$. The absence of a significant three-way interaction suggests that the asymmetrical coding effects were not different for the hue as opposed to the hedonic samples.

In addition, the pigeons performed significantly better overall on hedonic-sample trials than on hue-sample trials $[F(1,11)=21.79]$, and there was a significant sample type $\times$ common coding interaction $[F(1,11)=16.52]$. 
Simple main effect analyses were performed to identify the source of this interaction. The analyses indicated that although performance on $\mathrm{FH}$-sample trials was significantly lower than it was on F-sample trials $[F(1,11)=$ 46.33], performance on NFH-sample trials was not significantly lower than it was on NF-sample trials $[F(1,11)=$ 3.67].

Finally, in the three-way analysis there was also a significant sample type $\times$ delay interaction $[F(1,11)=$ 10.86]. Hue-sample and hedonic-sample delay functions tended to converge at the longest delay.

\section{Discussion}

In acquisition, there was differential responding to the two hue samples, depending on which hue was associated with the same comparison as was the $F$ sample. Paradoxically, there was more pecking to the NFH sample than there was to the FH sample. Given the hypothesis that the hue samples might be coded as hedonic samples, one might have expected the reverse to be true (i.e., more responding to the FH sample; see, e.g., Matzel, Held, \& Miller, 1988). Although it might be possible to account for these results in terms of some form of negative behavioral contrast (e.g., a hue that evokes a representation of food but is not accompanied by food may be relatively less appetitive than a hue that does not evoke such a representation), there may be a more parsimonious account of the difference in pecking the two hue samples. Because the hedonic-sample component of the task was generally acquired faster than the hue-sample component, it may be that early in training the pigeons tended to respond to one comparison when the sample was $\mathrm{F}$ and to the other comparison when the sample was not F (i.e., NF, FH, and NFH). Any such tendency would result in a high level of performance on NF sample trials as well as on NFH trials but would result in a lower level of performance on FH-sample trials. Thus, differences in hue-sample pecking may be related to differences in reinforcement probability associated with the two hues, rather than to the common coding of the hedonic and nonhedonic samples. Consistent with this hypothesis, performance on NFH trials was significantly better during training than was performance on $\mathrm{FH}$ trials $[F(1,11)=7.32]$. Furthermore, a Pearson productmoment correlation performed on the difference in performance on NFH- and $\mathrm{FH}$-sample trials in training (over all but the last three sessions) versus the difference between pecks to the NFH sample and to the FH sample (over the last three training sessions) was significant $[r(11)=.73]$.

When the pigeons were tested with delays, the typical nonparallel $F$ versus NF retention functions were found (see Colwill, 1984; Grant, 1991; Maki \& Hegvik, 1980; Sherburne \& Zentall, 1993b; Wilson \& Boakes, 1985). Moreover, a significant asymmetry was also found on hue-sample trials. This finding suggests that the pigeons in Experiment 1 commonly coded the two hue samples in terms of their hedonic counterparts. The fact that delay performance on hue-sample trials was generally less accurate than on hedonic-sample trials is consistent with the pigeons' use of $F$ representations as the basis for the common code. If the common code that relates $\mathrm{F}$ and $\mathrm{FH}$ samples involves a representation of the F sample (e.g. "means food"), more errors are likely to be made in using a derived code for the FH sample than in using a direct code for the $F$ sample. The relatively constant decrement in performance on $\mathrm{FH}$-sample trials as compared with $\mathrm{F}$ sample trials as a function of delay is consistent with the notion of such coding errors. It is also possible that the performance difference reflects differences in the saliency of the hedonic as opposed to the hue stimuli.

Although the delay data are consistent with such a common coding interpretation, two alternative accounts of the hue-sample asymmetry can be proposed (see Grant, 1991). First, as suggested earlier, it may be that the pigeons acquire this MTO matching task by first learning to choose one comparison when the sample is $F$, and to choose the other comparison whenever the sample is not $F$. With such a strategy a pigeon's matching accuracy would be about $75 \%$ correct, and accurate matching should be found on NFH trials but not on FH trials. With continued training, however, the pigeons may learn to code the FH sample and to associate it with the correct comparison. Thus, it may be that pigeons eventually learn to code F and FH samples independently, and that responding to the alternative comparison on NF and NFH trials is by default. Consistent with this hypothesis, performance in training was significantly more accurate overall on NFH trials than on FH trials.

According to the second alternative account, pigeons learn an association between each of the hue samples and its correct comparison. That is, pigeons may code $F$, FH, and NFH, and they may fail to code only NF. During 0 -sec delay training, a default response would be made only when codes for neither $F$, nor $F H$, nor NFH were present (i.e., on NF sample trials). In this case, the asymmetry in retention functions on hue-sample trials would result indirectly, from the default response made in the absence of a code. When delays are introduced, on $\mathrm{F}, \mathrm{FH}$, and NFH trials, as forgetting of the sample occurs, the tendency to respond by default should increase. With increasing retention interval, forgetting-produced default responding should result in a decrease in the level of performance on $\mathrm{F}$ - and $\mathrm{FH}$-sample trials, but relatively flat retention functions on both NF- and NFHsample trials. In the case of the NFH sample, a flat delay function would occur because the correct comparison would be chosen not only when the code was remembered, but also, by default, when it was not.

\section{EXPERIMENT 2}

The purpose of Experiment 2 was to clarify what was responsible for the asymmetrical retention functions found on the hue-sample trials in Experiment 1. To accomplish this, the transfer design described by Urcuioli 
et al. (1989) was used. Pigeons were trained on MTO DMTS with F, NF, FH, and NFH samples, with a procedure similar to that used in Experiment 1. They were then trained to respond to new comparison stimuli in the context of the hue samples alone. Finally, they were tested with the hedonic samples and the new comparisons, with tested associations either consistent or inconsistent with the presumed common codes. It was decided to use hue samples during interim training because previous research has shown that when interim training involves hedonic samples, and pigeons are transferred to nonhedonic samples, they show a strong bias to peck the comparison that in interim training was associated with the no-food sample (i.e., they correctly indicate that the sample was not food; Steirn, Jackson-Smith, \& Zentall, 1991; Urcuioli, DeMarse, and Zentall, 1994).

For purposes of comparison with the results of Experiment 1 , after the transfer test all pigeons were returned to the original 0 -sec delay MTO DMTS task and they were then tested with delays. The design of Experiment 2 is presented in Table 1 .

If the pigeons commonly code the samples associated with the same comparison, they should transfer at a higher level of performance in the consistent condition than in the inconsistent condition. On the other hand, if the F, FH, and NFH samples are independently coded, there should be little difference in performance under the two conditions. Finally, if the pigeons independently code $\mathrm{F}$ and $\mathrm{FH}$ samples and respond by default on both NF- and NFH-sample trials, pigeons in the consistent condition should transfer at a higher level of performance than those in the inconsistent condition only on NF-sample trials. In this case, the new default response acquired on NFH-sample trials in the interim training phase would be applicable to NF-sample trials in test.

In order to ensure that the hedonic-sample associations were acquired prior to the hue-sample associations and to encourage the development of the coding of hue samples as hedonic samples, all pigeons in Experiment 2 were trained on DMTS with F and NF samples alone, prior to MTO DMTS.

Table 1

Design of Experiment 2

\begin{tabular}{|c|c|c|c|c|c|}
\hline Group & $\begin{array}{c}\text { Initial } \\
\text { Training } \\
\end{array}$ & $\begin{array}{c}\text { MTO } \\
\text { Training } \\
\end{array}$ & $\begin{array}{c}\text { Interim } \\
\text { Training } \\
\end{array}$ & Test & $\begin{array}{c}\text { Delay } \\
\text { Test }\end{array}$ \\
\hline Consistent & $\begin{aligned} \mathrm{F} & \rightarrow \mathrm{C} \\
\mathrm{NF} & \rightarrow \mathrm{D}\end{aligned}$ & $\begin{aligned} \mathrm{F} & \rightarrow \mathrm{C} \\
\mathrm{NF} & \rightarrow \mathrm{D} \\
\mathrm{R} & \rightarrow \mathrm{C} \\
\mathrm{G} & \rightarrow \mathrm{D}\end{aligned}$ & $\begin{array}{l}\mathrm{R} \rightarrow \mathrm{B} \\
\mathrm{G} \rightarrow \mathrm{W}\end{array}$ & $\begin{aligned} \mathrm{F} & \rightarrow \mathrm{B} \\
\mathrm{NF} & \rightarrow \mathrm{W}\end{aligned}$ & $\begin{array}{r}\mathrm{F} \longrightarrow \mathrm{C} \\
\mathrm{NF} \longrightarrow \mathrm{D}\end{array}$ \\
\hline Inconsistent & $\begin{array}{r}\mathrm{F} \rightarrow \mathrm{C} \\
\mathrm{NF} \rightarrow \mathrm{D}\end{array}$ & $\begin{aligned} F & \rightarrow C \\
N F & \rightarrow D \\
R & \rightarrow D \\
G & \rightarrow C\end{aligned}$ & $\begin{array}{c}\mathrm{R} \rightarrow \mathrm{B} \\
\mathrm{G} \rightarrow \mathrm{W}\end{array}$ & $\begin{aligned} \mathrm{F} & \rightarrow \mathrm{B} \\
\mathrm{NF} & \rightarrow \mathrm{W}\end{aligned}$ & $\begin{array}{r}\mathrm{F} \longrightarrow \mathrm{C} \\
\mathrm{NF} \longrightarrow \mathrm{D}\end{array}$ \\
\hline
\end{tabular}

Note-Initial training involved food (F) and no-food (NF) samples and circle (C) and dot (D) comparisons. In many-to-one (MTO) training, red (R) and green (G) samples were added. In interim training, blue (B) and white (W) comparisons replaced the $C$ and D. Delay test involved mixed delays $(0,1,2$, and $4 \mathrm{sec})$.

\section{Method}

\section{Subjects and Apparatus}

The subjects were 12 White Carneaux pigeons similar to those used in Experiment 1. They were housed similarly to the pigeons in Experiment 1. The apparatus was similar to that used in Experiment 1 , with the exception that blue (B, Kodak Wratten filter No. 38a) and white (W, no filter) stimuli were added to the projectors behind the left and right response keys.

\section{Procedure}

Pretraining. All pigeons were pretrained to eat from the grain feeder and to peck $\mathrm{C}$ and $\mathrm{D}$ on the left and right response keys, as in Experiment 1.

Hedonic-sample DMTS training. On the following day, each pigeon began 0 -sec delay conditional discrimination training with $\mathrm{F}$ and NF samples, and C and D comparisons. In Experiment 2, mixed grain was used as both the $F$ sample and reinforcement for correct comparison responses, and the NF sample consisted of the feeder light alone. All other trial events were as they were in Experiment 1. All pigeons were trained on this task to a performance criterion of $90 \%$ correct for two consecutive sessions.

MTO DMTS training. On the following day, MTO DMTS training began. These sessions were the same as those in Experiment $\mathrm{l}$. For half of the pigeons, $\mathrm{R}$ served as the $\mathrm{FH}$ and $\mathrm{G}$ as the $\mathrm{NFH}$; for the remaining pigeons, $\mathrm{R}$ served as the $\mathrm{NFH}$ and $\mathrm{G}$ as the FH. The pigeons were trained on this task to a criterion of $90 \%$ correct for two consecutive sessions, or for a maximum of 200 sessions.

Interim training. Following MTO DMTS training, each pigeon was trained on a new task involving the $R$ and $G$ hue samples from MTO DMTS training and a new pair of comparisons (B and W). For all pigeons, responses to $\mathrm{W}$ following $\mathrm{R}$ samples and to $\mathrm{B}$ following $\mathrm{G}$ samples were reinforced. Each session of interim training involved only 64 trials, and each session was followed by a "refresher" session involving 32 trials of the MTO DMTS task. All pigeons were trained to a performance criterion of $90 \%$ correct for two consecutive sessions on the interim task as well as maintenance of at least $90 \%$ correct performance on each of the MTO DMTS sample types.

Transfer test. On the day following attainment of criterion, each pigeon was transferred to a new task involving $F$ and NF samples and $\mathrm{B}$ and $\mathrm{W}$ comparisons. For all pigeons, responses were reinforced to the $\mathrm{B}$ comparison following $\mathrm{F}$ samples, and to the $\mathrm{W}$ comparison following NF samples. Thus, for all pigeons that learned during MTO DMTS training to associate $\mathrm{R}$ with $\mathrm{C}$ and $\mathrm{G}$ with $D$, the reinforced associations in the transfer test were consistent with the presumed common codes, whereas for all pigeons that learned to associate $R$ with $D$ and $G$ with $C$, those associations were inconsistent with the presumed common codes. Each test session involved 64 trials, and training proceeded to the $90 \%$ correct criterion for two successive sessions.

Delay test. Following the transfer test, all pigeons were returned to the MTO DMTS task experienced earlier to reestablish their baseline levels of performance. As each pigeon returned to the criterion of $90 \%$ correct performance (for two consecutive sessions), it was exposed to mixed delay trials involving equal numbers of trials with delays of $0,1,2$, and $4 \mathrm{sec}$ placed between the offset of the sample and onset of the comparison stimuli. Each pigeon received ten 96-trial sessions of delay training.

\section{Results and Discussion}

\section{Training Phases}

Acquisition of DMTS with hedonic samples was rapid (mean $=12.1$ sessions). Acquisition of MTO DMTS was considerably more variable. Two of the pigeons failed to reach criterion in 200 sessions and were dropped from 
the study. It took the remaining pigeons an average of 76.0 sessions to reach criterion. Acquisition of the interim task involving the $\mathrm{R}$ and $\mathrm{G}$ samples and $\mathrm{B}$ and $\mathrm{W}$ comparisons took an average of 32.3 sessions.

\section{Transfer Test}

Overall performance of the consistent group was somewhat better than that of the inconsistent group, and the difference was relatively stable over the four test sessions for which there were data from all pigeons (mean difference $=10.36 \%$ correct); however, a one-way ANOVA performed on the transfer data failed to produce a significant difference in transfer performance $[F(1,8)=$ $1.63]$, because of considerable within-group variability. In earlier tests of common coding in pigeons, large individual differences have also been found. For example, Urcuioli et al. (1989) found that 3 of 12 pigeons showed no evidence of transfer indicative of common coding. Similarly, in a related experiment, Zentall et al. (1993) found that only pigeons that acquired MTO DMTS rapidly showed evidence of facilitation and interference effects attributable to common coding. In the present experiment, too, there was a significant negative Pearson product-moment correlation between rate of MTO DMTS acquisition and deviations from chance $(50 \%)$ test performance $[r(9)=-.572]$. In both the consistent and the inconsistent groups, pigeons that acquired the task quickly were the ones most likely to show transfer effects. The reason for this correlation may be that pigeons that commonly code the hues as the hedonic stimuli could learn their hue sample-correct comparison associations faster by using the already acquired associations between $F$ and NF samples and the same comparisons. For this reason, the pigeons were divided into fast and slow learners (median split) for purposes of data analysis. The data from fast and slow learners in the consistent and inconsistent groups are presented in Figure 2. The data are presented separately for each of the sample types.

A three-way mixed ANOVA was performed on the transfer data pooled over the first four transfer sessions, with compatibility (trial types consistent vs. inconsistent with presumed common codes), acquisition rate (fast vs. slow acquisition of MTO DMTS), and trial type (F vs. NF sample trials) as factors. The analysis indicated that there was a significant effect of compatibility $[F(1,6)=$ 7.11]. Pigeons for which the trial types in the transfer task were consistent with the presumed common codes acquired during MTO DMTS performed at a significantly higher level than those for which the trial types were inconsistent. There was also a significant effect of acquisition rate $[F(1,6)=6.35]$. Pigeons that acquired MTO DMTS faster also performed better on the transfer task, independently of condition. Although the effect of compatibility seemed to be greater for the fast learners than for the slow learners, the compatibility $\times$ acquisition rate interaction did not reach statistical significance $[F(1,6)=2.84]$. Finally, neither the effect of trial type

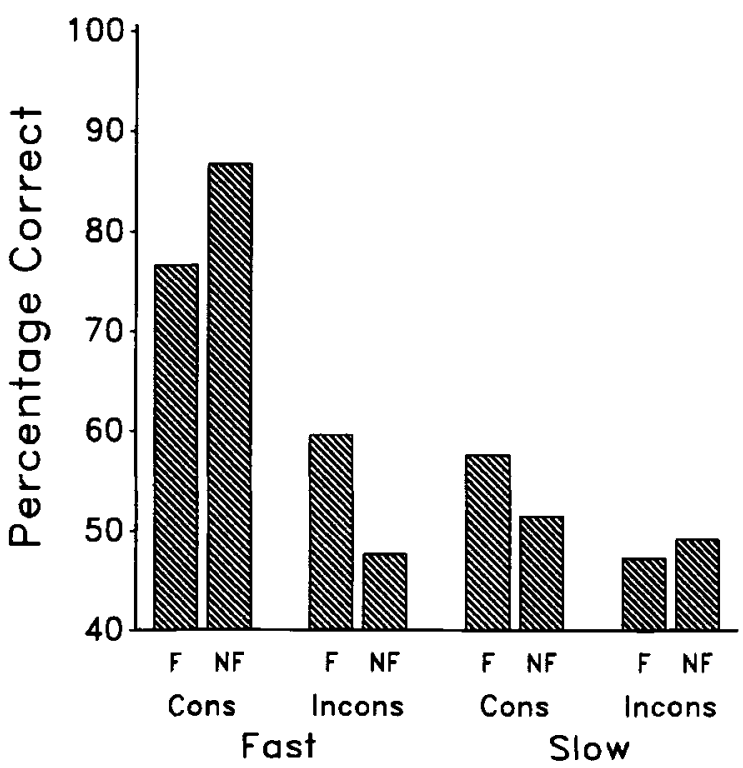

Figure 2. Experiment 2. Transfer performance, pooled over the first four transfer sessions, for pigeons that were fast versus slow to acquire original many-to-one delayed matching. For pigeons in the consistent group (Cons), the test trial types were consistent with the presumed common codes developed in original training. For pigeons in the inconsistent group (Incons) those trial types were inconsistent with the presumed common codes. Performance on food-sample trials (F) and no-food-sample trials (NF) are presented separately.

$(F<1)$ nor any of the interactions of trial type with the other factors were significant.

\section{Delay Test}

Data from the delay test confirmed the findings from Experiment 1. Performance on NF- and NFH-sample trials was at a high level over the range of delays tested, whereas performance on $\mathrm{F}$ - and $\mathrm{FH}$-sample trials was at a high level at the 0 -sec delay but dropped to below chance levels with increasing delays. Data from the delay test appear in Figure 3.

Comparison of these data with those from Figure 1 suggest the sample type asymmetries were more pronounced in Experiment 2 than in Experiment 1. It is likely that pretraining with the hedonic samples in Experiment 2 encouraged more of the pigeons to commonly code the hue samples as some representation of their hedonic counterparts. A three-way repeated measures ANOVA with sample type (hedonic vs. hue samples), common coding (F/FH vs. NF/NFH), and delay as factors indicated that there was significant common coding $[F(1,8)=9.47]$. Also, performance was significantly better on hedonic-sample trials than on hue-sample trials $[F(1,8)=25.68]$, and of course, there was a significant effect of delay $[F(3,24)=88.33]$. Of more importance was the significant common coding $X$ delay interaction $[F(3,24)=13.62]$. This interaction indicates that the F/FH versus NF/NFH functions were asymmetrical. A separate two-way analysis involving the hue sample data 


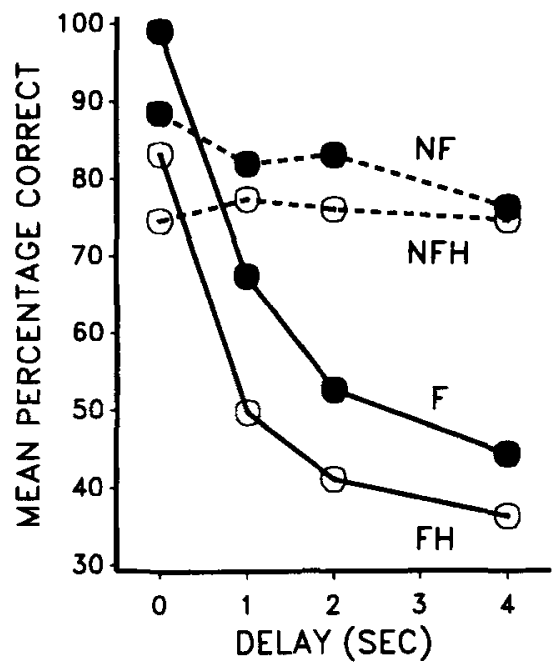

Figure 3. Experiment 2. Retention functions for each of the sample trial types in the many-to-one matching task: Food-sample trials (F), no-food-sample trials (NF), hue-sample trials for which the correct comparison was the same as on food-sample trials (FH), and hue-sample trials for which the correct comparison was the same as on no-food-sample trials (NFH).

alone yielded a significant sample type $\times$ delay interaction $[F(3,24)=10.70]$.

\section{GENERAL DISCUSSION}

The results of the present experiments extend the finding of common coding of samples associated with the same comparisons in MTO DMTS. In earlier research, evidence of common coding was reported for dimensions involving symmetrically coded samples (hues and shapes or line orientations). Here we have shown that common coding can be found between symmetrically coded hue samples and asymmetrically coded hedonic samples. Furthermore, using a transfer of training procedure in Experiment 2, we have shown that the common coding suggested by asymmetrical, hue-sample delay functions cannot be attributed simply to artifacts associated with default responding on no-food-sample trials, together with independent coding of some of the samples. Rather, the combined results from the transfer and delay tests suggest that pigeons can learn to code hue samples as representations of hedonic events when both are associated with correct responding to the same comparison stimulus (i.e., in an MTO mapping design).

The present results differ somewhat from the results of a similar experiment reported by Grant (1991, Experiment 2). In Grant's experiment, pigeons were trained on MTO DMTS involving hedonic ( $F$ and NF) and shape samples mapped onto hue comparisons. When Grant tested with delays, he failed to find the typical nonparallel F-sample versus NF-sample delay functions. Grant argued that the MTO mapping encouraged the pigeons to use prospective comparison codes (i.e., it was more efficient to remember which of two comparison stimuli was correct than to remember which of four samples had been presented). In the experiment reported here, the nonhedonic samples were hues (more salient and easier to discriminate than Grant's shapes-see Farthing et al., 1977), whereas the comparisons were shapes (less salient and harder to discriminate than Grant's hues). And as Zentall et al. (1989) reported, pigeons appear to code hue samples retrospectively regardless of the sampleto-comparison mapping ratio, whereas their coding strategy is sensitive to the sample-to-comparison mapping ratio when the samples are shapes. Thus, the presence of hue samples and easy-to-discriminate hedonic samples (as well as, perhaps, the presence of shape comparisons) in the present experiment produced results consistent with the retrospective common coding of hedonic and nonhedonic samples (see also Sherburne \& Zentall, 1993a).

The results of the present experiments are also consistent with results recently reported by Urcuioli et al. (1994). They found (in MTO DMTS with hedonic, hue, and differential-response samples; cf. Grant, 1982) asymmetrical retention functions not only on food- and no-food-sample trials, but also on hue- and responsesample trials. Thus, there was a consistency to the asymmetry across sample types that was related to the common comparison associations shared by those samples. In a follow-up experiment, Urcuioli et al. (1994, Experiment 2) provided independent transfer of control evidence that the hedonic, hue, and response samples that shared a common association were commonly coded.

The present results suggest that in combination, delay functions following MTO DMTS and transfer-of-training tests can help identify not only the nature of the coding process, but also the nature of the underlying codes themselves. These results thus add to the growing literature which indicates that the common coding of events that have been associated with the same stimuli or outcomes can play a significant role in learning by animals (e.g., Matzel et al., 1988; Urcuioli et al., 1989; Vaughan, 1988; Zentall et al., 1991).

\section{REFERENCES}

Berryman, R., Cumming, W. W., \& Nevin, J. A. (1963). Acquisition of delayed matching in the pigeon. Journal of the Experimental Analysis of Behavior, 9, 101-107.

ColWILL, R. M. (1984). Disruption of short-term memory for reinforcement by ambient illumination. Quarterly Journal of Experimental Psychology, 36B, 235-258.

Farthing, G. W., Wagner, J. M., Gilmour, S., \& Waxman, H. M. (1977). Short-term memory and information processing in pigeons. Learning \& Motivation, 8, 520-532.

Grant, D. S. (1982). Prospective versus retrospective coding of samples of stimuli, response, and reinforcers in delayed matching-tosample by pigeons. Learning \& Motivation, 13, 265-280.

GRANT, D. S. (1991). Symmetrical and asymmetrical coding of food and no-food samples in delayed matching in pigeons. Journal of Experimental Psychology: Animal Behavior Processes, 17, 186-193.

MAKJ, W. S., \& HEGVIK, D. K. (1980). Directed forgetting in pigeons. Animal Learning \& Behavior, 8, 567-574.

MAKI, W. S., Olson, D., \& REGo, S. (1981). Directed forgetting in pigeons: Analysis of cue functions. Animal Learning \& Behavior, 9 , $189-195$ 
Matzel, L. D., Held, F. P., \& Miller, R. R. (1988). Information and expression of simultaneous and backward associations: Implications for contiguity theory. Learning \& Motivation, 19, 317-344.

RoBerTs, W, A. (1972). Short-term memory in the pigeon: Effects of repetition and spacing. Journal of Experimental Psychology, 94, 7483.

Sherburne, L. M., \& Zentall, T. R. (1993a). Asymmetrical coding of food and no-food events by pigeons: Sample pecking versus food as the basis of the sample code. Learning \& Motivation, 24, 141155.

Sherburne, L. M., \& Zentall, T. R. (1993b). Coding of feature and no-feature events by pigeons performing a delayed conditional discrimination. Animal Learning \& Behavior, 21, 92-100.

Steirn, J. N., Jackson-Smith, P., \& Zentall, T. R. (1991). Mediational use of internal representations of food and no-food events by pigeons. Learning \& Motivation, 22, 353-365.

UrCuioli, P. J., DeMarse, T., \& Zentall T. R. (1994). Some properties of many-to-one matching with hue, response, and food samples: Retention and mediated transfer. Learning \& Motivation, 25, 175 200.

Urcuioli, P. J., Zentall, T. R., Jackson-Smith, P., \& Steirn, J. N. (1989). Evidence for common coding in many-to-one matching: $\mathrm{Re}$ tention, intertrial interference, and transfer. Journal of Experimental Psychology: Animal Behavior Processes, 15, 264-273.

VAughan, W., JR. (1988). Formation of equivalence sets in pigeons.
Journal of Experimental Psychology: Animal Behavior Processes, 14, 36-42.

Wilson, B., \& BoAkes, R. A. (1985). A comparison of the short-term memory performances of pigeons and jackdaws. Animal Learning \& Behavior, 13, 285-290.

ZENTALL, T. R. (1973). Memory in the pigeon: Retroactive inhibition in a delayed matching task. Bulletin of the Psychonomic Society, 1, 126-128.

Zentall, T. R., Sherburne, L. M., \& Urculoli, P. J. (1993). Common coding by pigeons in a many-to-one delayed matching task as evidenced by facilitation and interference effects. Animal Learning \& Behavior, 21, 233-237.

Zentall, T. R., Steirn, J. N., Sherburne, L. M., \& Urcuioli, P. J. (1991). Common coding in pigeons assessed through partial versus total reversals of many-to-one conditional and simple discriminations. Journal of Experimental Psychology: Animal Behavior Processes, 17, 194-201.

Zentall, T. R., Urcuioli, P. J., Jagielo, J. A., \& JaCkson-Smith, P. (1989). Interaction of sample dimension and sample-comparison mapping on pigeons' performance of delayed conditional discriminations. Animal Learning \& Behavior, 17, 172-178.

(Manuscript received February 19, 1993; revision accepted for publication July 30, 1994.) 